\title{
Cognitive Medium Access: A Protocol for Enhancing Coexistence in WLAN Bands
}

\author{
Stefan Geirhofer and Lang Tong \\ School of Electrical and Computer Engineering \\ Cornell University, Ithaca, NY 14853 \\ Email: $\{$ sg355, 1t35\}@ cornell.edu
}

\author{
Brian M. Sadler \\ Army Research Laboratory \\ Adelphi, MD 20783-1197 \\ Email: bsadler@arl.army.mil
}

\begin{abstract}
In this paper we propose Cognitive Medium Access (CMA), a protocol aimed at improving coexistence with a set of independently evolving WLAN bands. A time-slotted physical layer for the cognitive radio is considered and CMA is derived based on experimental models. By recasting the problem as a Constrained Markov Decision Process (CMDP), throughput is optimized while keeping interference below some given constraint. The optimal control policy is obtained via linear programming. In addition, we show that optimal CMA admits structured solutions which are computationally less expensive and allow further insight into the problem. Numerical results are presented for typical coexistence setups and show a significant performance improvement.
\end{abstract}

\section{INTRODUCTION}

Wireless communications has become ubiquitous in today's society leading to a dense allocation of relevant frequency bands. Actual measurements [1], however, reflect that most of these frequency bands are vastly underutilized because they are statically assigned to a single licensee. The resulting scarcity of available frequency bands confines many consumer applications to the unlicensed ISM bands which in turn are becoming increasingly crowded. Wireless communications in these bands is often limited by mutual interference.

Recent advances in software-defined radio have sparked interest in resolving this paradox by dynamically accessing spectrum. In this paper, we consider hierarchical setups [2], where the cognitive system is designed such that no or only insignificant interference is generated toward the primary user. Specifically, we identify the primary user with a set of parallel WLAN channels and consider a time-slotted physical layer for the cognitive radio.

A hierarchical approach mandates that orthogonality be maintained between both systems. In our work this is guaranteed in the time domain, by predicting the WLAN's medium access based on a stochastic model [3]. Interference is avoided by reusing idle periods between bursty WLAN transmissions. The cognitive radio's hopping sequence is altered such that it preferably hops to bands not currently used by the WLAN.

\footnotetext{
${ }^{1}$ This paper was prepared though collaborative participation in the Communications and Networks Consortium sponsored by the U.S. Army Research Laboratory under the Collaborative Technology Alliance Program, Cooperative Agreement DAAD19-01-2-0011. The U.S. Government is authorized to reproduce and distribute reprints for Government purposes notwithstanding any copyright notation thereon.
}

The cognitive radio designed in this paper could be viewed as a "smart" Bluetooth device. This will enable us to compare the performance of our schemes with this benchmark.

\section{A. Main contribution}

The paper's main contribution is the proposition of Cognitive Medium Access (CMA), a protocol that maximizes the throughput of the cognitive radio while satisfying interference constraints. We consider two metrics, namely (i) a cumulative interference constraint (CIC) reflecting the number of slot collisions per unit time, and (ii) a packet error rate constraint (PERC), limiting the number of WLAN packet errors due to the interferer. Both constraints are enforced based on a WLAN prediction model previously proposed [3].

CMA is derived mathematically by formulating the problem within the framework of Constrained Markov Decision Processes (CMDPs). The optimal control of the CMDP can be obtained via linear programming [4]. We briefly state the standard solution technique. Moreover, we show that structured solutions exist for both types of interference constraints, which facilitate implementation and allow for additional insight into the problem.

\section{B. Related work}

CMA is relevant to both the cognitive radio and the coexistence community. Among the first to consider time-domain dynamic spectrum access is [5], deriving access schemes based on identical slot structures for primary and secondary system. Modeling the medium access of unslotted systems such as WLAN has been considered in [3], [6] and led to a semiMarkov model. A continuous-time Markov chain (CTMC) approximation has been considered in [7] together with a periodic sensing approach.

Within the coexistence framework, our problem falls into the category of adaptive frequency hopping. Such methods are of particular interest to improve WLAN/Bluetooth coexistence [8]. However, in contrast to existing schemes, which classify channels based on feedback from higher-layers, our approach is able to capture the dynamic interference from the WLAN by pursuing a model-based design approach.

The rest of this paper is organized as follows. In Sec. II we introduce the system setup and summarize our experimental studies. In Sec. III we derive CMA within the framework of 


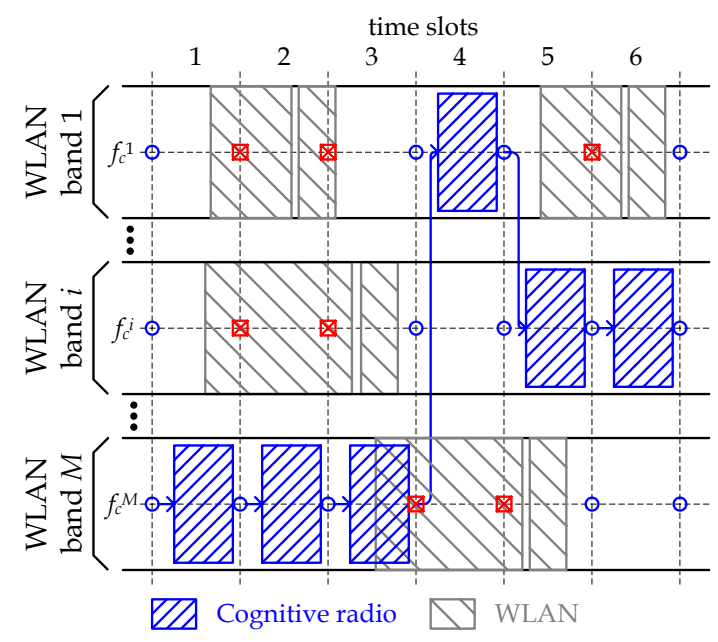

Fig. 1. System setup. The cognitive radio is time-slotted and uses either FH or DSSS (shown above). Circles (squares) denote and idle (busy) sensing result. A collision is shown in slot 3 .

CMDPs. The structure of the optimal policy is obtained in Sec. IV. Numerical results are given in Sec. V.

\section{System Setup}

In this paper we consider a set of $M$ parallel WLAN bands as depicted in Fig. 1. The bands evolve independently according to the standard [9]. WLAN implements the CSMA/CA protocol for multi user access and is thus an unslotted system.

\section{A. Physical layer design}

The design of the cognitive radio focuses on the timeslotted system depicted in Fig. 1. Specifically, we consider two different setups, namely (i) a frequency-hopping (FH) setup, and (ii) a direct-sequence spread spectrum (DSSS) physical layer.

The FH setup is similar to standard Bluetooth. Transmissions are initiated in narrowband channels, with $N$ channels overlapping with each of the $M$ WLAN bands. Mutual interference is reduced since the WLAN has some inherent robustness to narrowband interference. The DSSS setup is similar to 802.11b WLAN [10] but uses a different spreading code to enable coexistence. Both setups are equivalent in terms of formulating CMA, since we only design optimal hopping across the $M$ WLAN bands [11].

Choosing a slotted system is a natural approach for limiting interference. By employing a sense-before-transmit strategy collisions can occur only if the primary user becomes active in the current slot. Interference can thus be limited based on predicting the WLAN's access behavior.

\section{B. Operation}

The operation of the cognitive system is shown in Fig. 2. At the beginning of every slot a spectrum sensor determines whether the medium is busy or idle. We assume the frontend is powerful enough to observe the state of all $M$ bands, leading to a fully-observable scenario. If only a single band can be sensed at a time the problem becomes significantly more

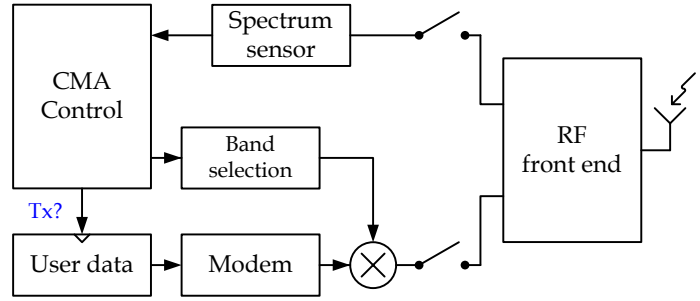

Fig. 2. Block diagram of the cognitive radio's operation.

complex. We extend our work to this partially-observable case in [11].

Based on the sensing result, the CMA controller determines whether to initiate a transmission, and if yes in which band. We will show that optimal CMA leads to a randomized control policy: based on the current sensor reading a biased coin flip determines whether or not to transmit. We assume perfect sensing throughout the paper.

In the scope of this paper we shall focus on a single master/slave architecture for the cognitive system. We assume that the sensing results of all nodes are the same, and achieve a joint hopping pattern by sharing a random seed. Additional information can be encoded in higher-layers, similar to Bluetooth [12].

\section{Empirical interference models}

The interaction between both systems has been characterized in an experimental study [11], [13]. Specifically, we evaluated (i) the impact on the WLAN's carrier sensing and (ii) determined the probability of a collision leading to a packet error. We stress that both models are based on actual measurements at $2.4 \mathrm{GHz}$.

The cognitive radio may impact the WLAN's carrier sensing, if it is mistaken as another WLAN station. Clearly this would alter the medium access and violate our hierarchial approach. Our measurement results show, however, that for typical deployment scenarios, the cognitive radio's transmissions appear transparent [13].

While we anticipate no impact on the WLAN's carrier sensing, collisions between both systems cause WLAN packet errors. We have evaluated by measurement the probability of a collision leading to a packet error [13]. Our results show a strong dependence on the signal-to-interference ratio (SIR) at the receiver. In the scope of this paper we consider the worst-case assumption that every collision inevitably leads to a packet error and refer to [13] for detailed measurement results.

\section{Cognitive Medium Access}

In this section we derive CMA based on a stochastic model of WLAN's medium access which enables us to constrain the number of slot collisions. We briefly review the model, formulate the problem as a CMDP, and state the standard solution technique.

\section{A. Semi-Markov model and CTMC approximation}

CMA exploits idle periods that remain between bursty WLAN transmissions. In order to be able to predict the 
WLAN's behavior we need to stochastically model the idle and busy durations of the channel. Based on empirical data gathered via a sensing testbed we showed that a semi-Markov model (SMM) with a mixture fit provides for an accurate characterization [6]. The fit was verified based on statistical measures.

While the SMM is accurate, it is complicated to derive access schemes based on it. To improve tractability we consider an exponential approximation,

$$
\text { idle: } F_{0}(t)=1-e^{-\lambda t}, \quad \text { busy: } F_{1}(t)=1-e^{-\mu t},
$$

which leads to a continuous-time Markov chain (CTMC) with parameters $\lambda$ and $\mu$. The memoryless property significantly simplifies the derivation. Moreover, although the exponential fit is not strictly validated by statistical measures of fit, it shows a good match with the empirical distribution. We have evaluated the robustness of the CTMC approximation and achieved promising results. We refer to [11] for more details.

\section{B. Decision-theoretic formulation}

We proceed by defining the CMDP. Let each of the $M$ WLAN bands evolve according to a CTMC $\left\{X_{i}(t), t \geq 0\right\}$ with states ' 0 ' (idle) and ' 1 ' (busy). Note that this is a continuous time random process with holding times as in (1). The cognitive radio senses these bands at the beginning of every slot, inducing the discrete time processes $\left\{Y_{i}[k], k \in\right.$ $\left.\mathbb{Z}^{+}\right\}$for each band. For notational convenience collect all sensing results in the vector-valued process

$$
\mathbf{Y}[k]=\left[Y_{1}[k], \ldots, Y_{M}[k]\right]^{T} .
$$

Transition behavior: The transition behavior and stationary distribution of this chain can easily be obtained by exploiting the independence of the bands and well known properties of CTMCs. The stationary distribution of the $i$-th band evaluates to

$$
\eta_{0}^{(i)}=\frac{\mu_{i}}{\lambda_{i}+\mu_{i}}, \quad \eta_{1}^{(i)}=\frac{\lambda_{i}}{\lambda_{i}+\mu_{i}}
$$

and the transition matrix [14, p.391] is given by

$$
\mathbf{P}^{(i)}=\frac{1}{\lambda_{i}+\mu_{i}}\left[\begin{array}{ll}
\mu_{i}+\lambda_{i} e^{-\left(\lambda_{i}+\mu_{i}\right) t} & \lambda_{i}-\lambda_{i} e^{-\left(\lambda_{i}+\mu_{i}\right) t} \\
\mu_{i}-\mu_{i} e^{-\left(\lambda_{i}+\mu_{i}\right) t} & \lambda_{i}+\mu_{i} e^{-\left(\lambda_{i}+\mu_{i}\right) t}
\end{array}\right] .
$$

Control dimension: Based on the sensing results, the CMA controller determines in which (if any) channel to transmit. Its actions are represented by the set $\mathbb{A}=\{0,1, \ldots, M\}$, where $a=0$ denotes that no transmission takes place and $a \geq 0$ denotes that a transmission is initiated across channel $a$.

Reward and cost structure: Throughput optimization subject to the interference constraint is based on expected immediate rewards/costs that are accrued/incurred based on choosing action $a$ in state $\mathbf{y}$ along the sample path [11]. We accrue a unit reward for a successful transmission which leads to the expected immediate reward

$$
r(\mathbf{y}, a)=\left\{\begin{array}{cc}
1_{\left[y_{a}=0\right]} e^{-\lambda_{a} T_{s}}, & a \geq 1 \\
0, & a=0
\end{array} .\right.
$$

We consider two different interference metrics. First, the cumulative interference constraint (CIC) limits the number of slot collisions per unit time. This leads to the immediate cost

$$
d_{c}(\mathbf{y}, a)=\left\{\begin{array}{cl}
1-e^{-\lambda_{a} T_{s}} & \text { if } y_{a}=0, a \geq 1 \\
1 & \text { if } y_{a}=1, a \geq 1 . \\
0 & \text { if } a=0
\end{array} .\right.
$$

However, the above formulation does not condition on the WLAN's traffic density. We incorporate this dependence by imposing packet error rate constraints (PERCs) for each WLAN band,

$$
d_{p}(\mathbf{y}, a)=\left\{\begin{array}{cl}
\frac{\left(\lambda_{a}+\mu_{a}\right)\left(1-e^{-\lambda_{a} T_{s}}\right)}{\mu_{a} \lambda_{a} T_{s}} & \text { if } y_{a}=0, a \geq 1 \\
1 & \text { if } y_{a}=1, a \geq 1 \\
0 & \text { if } a=0
\end{array} .\right.
$$

The PERCs corresponds to the average percentage of packet errors due to the cognitive radio's interference.

CMDP formulation: Finally, we need to define optimization criteria based on the immediate rewards/costs. The objective is to maximize the expected average reward

$$
J(\pi)=\lim _{N \rightarrow \infty} \frac{1}{N} \sum_{t=1}^{N} \mathbb{E}^{\pi} r\left(\mathbf{Y}_{t}, A_{t}\right)
$$

subject to a CIC,

$$
D_{c}(\pi)=\lim _{N \rightarrow \infty} \frac{1}{N} \sum_{t=1}^{N} \mathbb{E}^{\pi} d_{c}\left(\mathbf{Y}_{t}, A_{t}\right) \leq \alpha,
$$

or subject to PERCs for each band $1 \leq i \leq M$

$$
D_{p}^{(i)}(\pi)=\lim _{N \rightarrow \infty} \frac{1}{N} \sum_{t=1}^{N} \mathbb{E}^{\pi} 1_{\left[A_{t}=i\right]} d_{p}\left(\mathbf{Y}_{t}, A_{t}\right) \leq \alpha_{i} .
$$

\section{Solution strategy}

The CMDP formulation (8)-(10) can be solved via linear programming [4]. It is well known that the optimal control of a CMDP corresponds to a randomized stationary policy [15]. This means that in state $\mathbf{y}$, the controller chooses randomly among a set of actions $\mathbb{A}(\mathbf{y})$. Note that in practice this is easily implemented by flipping biased coins.

The standard solution technique uses the long-run frequency of state-action pairs, $\rho(\mathbf{y}, a)$, to express the objective function and the constraints. This leads to an equivalent linear programming formulation (see [4] for details).

Theorem [4, p.38]: The linear program

$$
\max _{\rho(\mathbf{y}, a)} \sum_{\mathbf{y} \in \mathbb{X}} \sum_{a \in \mathbb{A}(\mathbf{y})} \rho(\mathbf{y}, a) r(\mathbf{y}, a)
$$

subject to

$$
\sum_{\mathbf{y} \in \mathbb{X}} \sum_{a \in \mathbb{A}(\mathbf{y})} \rho(\mathbf{y}, a) d_{i}(\mathbf{y}, a) \leq \alpha_{i}, 1 \leq i \leq M,
$$

where $\rho(\mathbf{y}, a) \in \mathbb{Q}$ and

$$
\mathbb{Q}=\left\{\begin{array}{l}
\rho(\mathbf{y}, a), \mathbf{y} \in \mathbb{X}, a \in \mathbb{A}(\mathbf{y}): \\
\sum_{\mathbf{y} \in \mathbb{X}} \sum_{a \in \mathbb{A}(\mathbf{y})} \rho(\mathbf{y}, a)\left(\delta_{\mathbf{y}}(\mathbf{x})-P_{\mathbf{x} a \mathbf{y}}\right)=0 \\
\sum_{\mathbf{y} \in \mathbb{X}} \sum_{a \in \mathbb{A}(\mathbf{y})} \rho(\mathbf{y}, a)=1, \rho(\mathbf{y}, a) \geq 0
\end{array}\right\}
$$


is equivalent to the CMDP formulations (8)-(10). $\square$ Based on $\rho(\mathbf{y}, a)$ the probability of choosing action $a$ in $\mathbf{y}$ is

$$
w_{\mathbf{y}}(a)=\frac{\rho(\mathbf{y}, a)}{\sum_{j \in \mathbb{A}(y)} \rho(\mathbf{y}, j)} .
$$

\section{Structured Policies}

While optimal policies can be computed using the LP (11)-(13), we show that our specific problem setup leads to structured solutions. This facilitates implementation and allows further insight into the problem.

\section{A. Cumulative Interference Constraint (CMA-CIC)}

Without loss of generality assume that the channels are ordered with respect to their mean idle periods, $\lambda_{1} \leq \cdots \leq$ $\lambda_{M}$. For a given CIC level $\alpha$ we show that it is optimal to transmit in the first $k(\alpha)$ channels only.

Two important observations in (11)-(13) contribute to the special structure. First, note that $d_{c}(\mathbf{y}, a)=1-r(\mathbf{y}, a)$ for all state-action pairs. Second, the optimal policy is not unique. To see this, define sets $\mathbb{X}_{i}$, which include all states for which channel $i$ is idle

$$
\mathbb{X}_{i}=\left\{\mathbf{x} \in \mathbb{X}: x_{i}=0\right\}, 1 \leq i \leq M .
$$

States $\mathbf{x}, \mathbf{y} \in \mathbb{X}_{i}$ offer the same reward at the same cost for transmitting across channel $i$ and are thus equivalent.

This leads to the following threshold policy (cf. Fig. 3). After observing the sensing result $\mathbf{y}$ for all channels, define $i$ as the idle channel with the smallest index. Transmit in this channel with certainty if $i<k(\alpha)$ and do not transmit if $i>k(\alpha)$. If $i=k(\alpha)$ randomize, i.e., transmit with a certain probability $w_{i}$.

The threshold $k(\alpha)$ is obtained based on the CIC level $\alpha$. Transmissions can only occur in the first idle channel and thus the maximum interference level from channel $i$ amounts to

$$
\xi_{i}=\sum_{\mathbf{y} \in \mathbb{X}_{i}} 1_{\left[y_{j}=1, \forall j<i\right]} \rho(\mathbf{y}, i) .
$$

The threshold $k(\alpha)$ is the smallest $k$ such that

$$
\xi_{1}+\cdots+\xi_{k}>\alpha .
$$

By definition of the $\left\{\xi_{i}\right\}$ we obtain the randomization $w_{i}$ as

$$
w_{k}=\frac{\alpha-\xi_{k(\alpha)-1}}{\xi_{k(\alpha)}-\xi_{k(\alpha)-1}} .
$$

\section{B. Packet Error Rate Constraints (CMA-PERC)}

In the case of PERCs, we have separate constraints for each band. Since $r(\mathbf{y}, a) \geq 0$ the maximum reward would be achieved if all constraints could be made tight. This may not be possible, however, because we can only transmit in one channel per slot. Even if we transmitted in every slot, not all PERCs can be made tight.

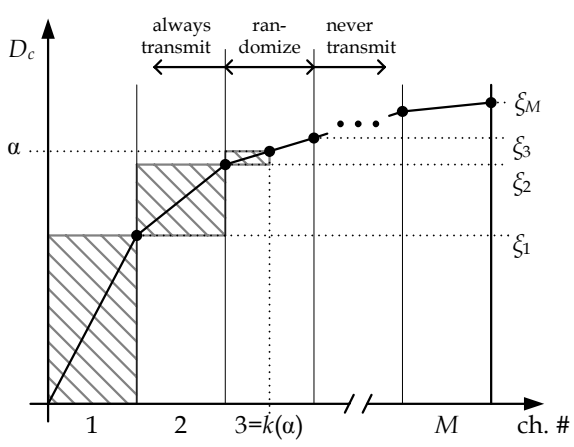

Fig. 3. Threshold solution for CMA-CIC.

In practical scenarios, however, the constraints will usually be tight enough to limit the transmission behavior. Specifically, if the following sufficient condition is met for all channels,

$$
\xi_{a}=\sum_{x \in \mathbb{X}} \frac{1_{\left[x_{a}=0\right]} \eta_{\mathbf{x}}}{\sum_{l=1}^{M} 1_{\left[x_{l}=0\right]}} \geq \frac{\alpha_{a}}{d_{a}}, \forall a \in \mathbb{A},
$$

where

$$
d_{a}=\frac{\left(\lambda_{a}+\mu_{a}\right)\left(1-e^{-\lambda_{a} T_{s}}\right)}{\mu_{a} \lambda_{a} T_{s}} .
$$

the optimal policy has a very simple structure [11].

If (19) is met, $\sum_{i=1}^{M} w_{\mathbf{y}}(i) \leq 1$, and the constraints can be made tight. We can thus transmit in band $i$ with probability

$$
w_{\mathbf{y}}(a)=\frac{\alpha_{a} /\left(d_{a} \xi_{a}\right)}{\sum_{l=1}^{M} 1_{\left[y_{l}=0\right]}} .
$$

\section{Numerical Results}

In this section we present numerical results for the CMA protocol and compare its performance to a blind hopping scheme such as standard Bluetooth. Furthermore, we show a practical example for the threshold policy introduced in Sec. IV.

\section{A. Simulation parameters}

As typical in coexistence setups, the performance of CMA depends on the dynamic behavior of the WLAN. Our numerical analysis thus evaluates CMA's throughput and interference behavior for varying WLAN traffic load. The WLAN traffic load is indexed by the parameter $\sigma$, where small values denote light utilization, while $\sigma$ close to one denotes heavy traffic load. The numerical results are obtained via simulations using the CTMC approximation. Its parameters, however, were extracted from an actual 802.11b system [6] and can be found in detail in [13]. The remaining simulation parameters were chosen to mimic a typical Bluetooth/WLAN coexistence setup [11].

\section{B. Throughput and interference of CMA}

The throughput and interference of CMA are shown in Fig. 4 for varying WLAN traffic load $\sigma$. Note that Fig. 4 shows the throughput, the cumulative interference, and the packet error rate for each scheme separately.

We compare the performance of CMA to a blind hopping scheme similar to standard Bluetooth. This oblivious interferer 

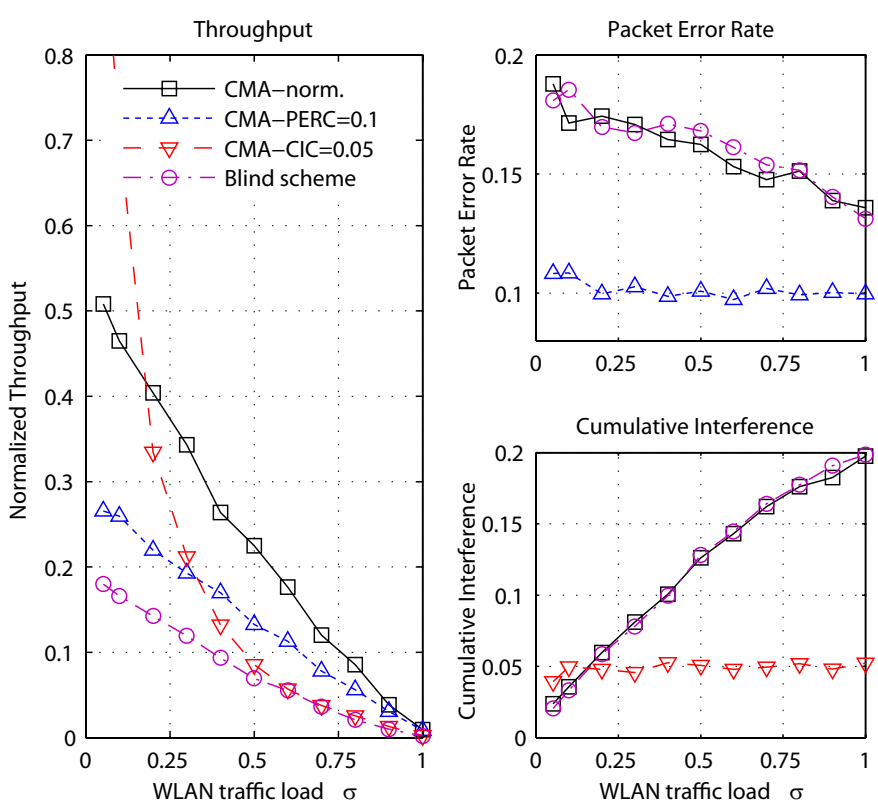

Fig. 4. Comparing CMA with a blind scheme. CMA-norm is designed to have the same PERC as the blind transmitter. CMA-PERC and CMA-CIC impose the specified constraint across $\sigma$.

transmits in a randomly selected channel in every fifth slot. We observe a small throughput and a packet error rate of about 15-20\%. CMA significantly outperforms this blind scheme. In order to provide a fair comparison we chose the constraints $\alpha$ such that CMA has the same packet error rate as the oblivious transmitter. This leads to a throughput increase by a factor of 2.5. The packet error rate decreases with $\sigma$ since, at low $\sigma$, fewer collisions occur in total, but also fewer WLAN packets are transmitted.

We have also plotted the performance for a constant PERC of $\alpha_{i}=0.1 \forall i$ and CIC of $\alpha=0.05$, respectively. The corresponding curves are shown in the same plot and outperform the oblivious transmitter's throughput while offering smaller interference at the same time. Note that at least for small $\sigma$, constraining the packet error rate is more restrictive than limiting the average number of slot collisions.

\section{Threshold Policy}

Second, we evaluate the CMA throughput with respect to increasing interference constraints $\alpha\left(\alpha_{i}=\alpha \forall i\right.$ for PERC). For CMA-CIC we can expect to observe $M$ piecewise linear segments due to the threshold policy. Indeed, Fig. 5 shows that the channel with smallest $\lambda$ is predominantly used and by enlarging the plot we can identify the $M$ segments. For CMA-PERC, we observe a linear increase with $\alpha$.

\section{Vi. CONCLUSIONS}

In conclusion we have proposed CMA, a cognitive medium access protocol that enhances coexistence with a set of parallel WLAN bands. CMA's derivation is based on an empirical model which predicts the WLAN's bursty medium access. Access strategies were derived based on a CMDP formulation and linear programming. We identified structured policies

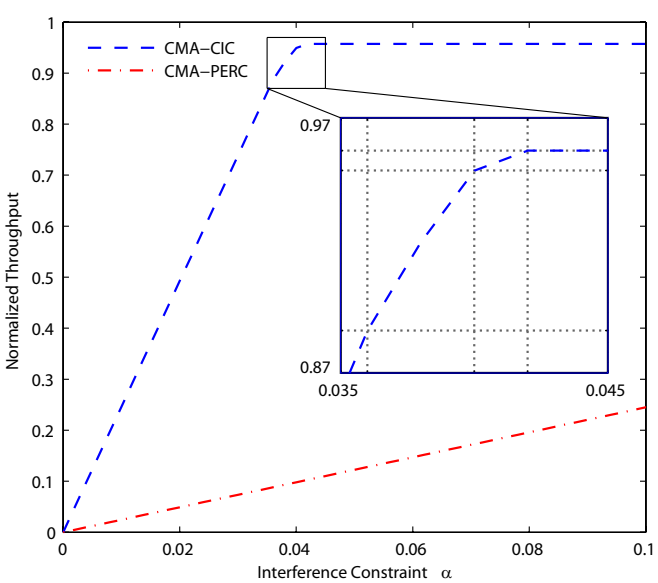

Fig. 5. Throughput vs. interference constraints. Thresholds for CMA-CIC are indicated with dotted lines.

that allow further insight into the problem. The numerical assessment showed that a significant performance gain can be expected in realistic coexistence scenarios.

\section{REFERENCES}

[1] D. Čabrić, S. M. Mishra, D. Willkomm, R. Broderson, and A. Wolisz, "A Cognitive Radio Approach for Usage of Virtual Unlicensed Spectrum," in Proc. IST Mobile Wireless Communications Summit, 2005.

[2] Q. Zhao and B. M. Sadler, "Dynamic Spectrum Access: Signal Processing, Networking, and Regulatory Policy," IEEE Signal Processing Mag., vol. 55, no. 5, pp. 2294-2309, May 2007.

[3] S. Geirhofer, L. Tong, and B. M. Sadler, "Dynamic Spectrum Access in the Time Domain: Modeling and Exploiting Whitespace," IEEE Commun. Mag., vol. 45, no. 5, pp. 66-72, May 2007.

[4] E. Altman, Constrained Markov Decision Processes. Chapman \& Hall/CRC, 1999.

[5] Q. Zhao, L. Tong, A. Swami, and Y. Chen, "Decentralized Cognitive MAC for Opportunistic Spectrum Access in Ad Hoc Networks: A POMDP Framework," IEEE J. Select. Areas Commun., vol. 25, no. 3, pp. 589-600, Apr. 2007.

[6] S. Geirhofer, L. Tong, and B. M. Sadler, "Dynamic Spectrum Access in WLAN Channels: Empirical Model and Its Stochastic Analysis," in Proc. First International Workshop on Technology and Policy for Accessing Spectrum, 2006.

[7] Q. Zhao, S. Geirhofer, L. Tong, and B. M. Sadler, "Optimal Dynamic Spectrum Access via Periodic Channel Sensing," Proc. IEEE Wirless Communications and Networking Conference (WCNC), Mar. 2007.

[8] IEEE Computer Society, "IEEE Std 802.15.2-2003, Coexistence of Wireless Personal Area Networks with Other Wireless Devices Operating in Unlicensed Frequency Bands," Aug. 2003.

[9] ANSI/IEEE Standard 802.11, 1999 Edition (R2003), "Wireless LAN Medium Access Control (MAC) and Physical Layer (PHY) Specifications," IEEE/SA Standards Board, Tech. Rep., 1999.

[10] ANSI/IEEE Standard 802.11b-1999 (R2003), "Wireless LAN Medium Access Control (MAC) and Physical Layer (PHY) specifications: Higher-Speed Physical Layer Extension in the $2.4 \mathrm{GHz}$ band," IEEE SA Standards Board, Tech. Rep., 1999.

[11] S. Geirhofer, L. Tong, and B. M. Sadler, "Cognitive Medium Access: Constraining Interference Based on Experimental Models," submitted to IEEE Journal on Selected Areas in Communications, Mar. 2007.

[12] Bluetooth Special Interest Group, "Specification of the Bluetooth System," Nov. 2004.

[13] S. Geirhofer, L. Tong, and B. M. Sadler, "Measurement-Based Models for Cognitive Medium Access in WLAN Bands," Cornell University, Adaptive Communications and Signal Processing Group (ACSP), Technical Report ACSP-TR-02-07-02, Feb. 2007. [Online]. Available: http://acsp.ece.cornell.edu/papers/ACSP-TR-02-07-02.pdf

[14] S. I. Resnick, Adventures in Stochastic Processes. Birkhäuser, 1992.

[15] M. L. Puterman, Markov Decision Processes. Discrete Stochastic Dynamic Programming. John Wiley \& Sons, Inc., 1994. 